\title{
Diagnostic Value of Serum Procollagen III N-Terminal Peptide for Liver Fibrosis in Infantile Cholestasis
}

\author{
Yingcan Wang ${ }^{1+}$, Weihua Pan ${ }^{2+}$, Dongying Zhao ${ }^{1}$, Yan Chen ${ }^{1}$, Xuting Chen ${ }^{1}$ and \\ Hongping Xia ${ }^{1 *}$ \\ ${ }^{1}$ Department of Neonatology, Xinhua Hospital, Shanghai Jiao Tong University School of Medicine, Shanghai, China, \\ ${ }^{2}$ Department of Pediatric Surgery, Xinhua Hospital, Shanghai Jiao Tong University School of Medicine, Shanghai, China
}

OPEN ACCESS

Edited by:

Pietro Vajro,

University of Salerno, Italy

Reviewed by:

Tudor Lucian Pop,

Iuliu Haţieganu University of Medicine and Pharmacy, Romania

Björn Fischler,

Karolinska University

Hospital, Sweden

${ }^{*}$ Correspondence:

Hongping Xia

xiahongping@xinhuamed.com.cn

tThese authors have contributed equally to this work

Specialty section:

This article was submitted to Pediatric Gastroenterology,

Hepatology and Nutrition,

a section of the journal

Frontiers in Pediatrics

Received: 09 January 2020 Accepted: 10 March 2020

Published: 31 March 2020

Citation:

Wang Y, Pan W, Zhao D, Chen Y, Chen $X$ and Xia H (2020) Diagnostic

Value of Serum Procollagen III $N$-Terminal Peptide for Liver Fibrosis in Infantile Cholestasis.

Front. Pediatr. 8:131.

doi: 10.3389/fped.2020.00131
Background: Several non-invasive markers have been reported as being effective for the assessment of fibrosis in adults with chronic viral hepatitis. The infantile liver is more susceptible to cholestasis, and it is important to promptly evaluate liver fibrosis to guide the clinical treatment. However, the clinical value of these markers in infants with cholestasis remains unknown.

Aim: To investigate the correlation between serum laminin $(\llcorner N)$, hyaluronic acid $(\mathrm{HA})$, procollagen III N-terminal peptide (PIIINP) level, and liver fibrosis stage in infants with cholestasis.

Methods: One hundred and thirty-seven term infants with cholestasis were included. Laparoscopic exploration and cholangiography were performed to diagnose or rule out biliary atresia. Serum LN, HA, and PIIINP were measured prior to laparoscopic exploration. Liver biopsy was performed for all patients. Liver fibrosis was staged on a five-point scale (FO-F4) according to the METAVIR scoring system. The correlation between serum markers and liver fibrosis stage was assessed. A receiver operator characteristic analysis was performed to determine the accuracy of serum markers for predicting the liver fibrosis stage.

Results: Serum PIIINP and HA were positively correlated with liver fibrosis stage ( $r=0.622, P<0.001$, and $r=0.41, P<0.001$, respectively). There was no significant correlation between serum $L N$ and liver fibrosis stage $(P>0.05)$. Serum aspartate aminotransferase, total bilirubin, direct bilirubin, and PIIINP were independently correlated with the fibrosis stage on multivariate ordinal regression analysis. Receiver operating curve $(\mathrm{ROC})$ analysis showed that serum PIIINP was the most effective for the diagnosis of fibrosis grade. The area under the ROC curves (AUROCs) for serum PIIINP for diagnosing fibrosis stages $\geq F 1, \geq F 2$, $\geq F 3$, and F4 (cirrhosis) were 0.843, 0.789, 0.82, and 0.891, respectively. The cut-off serum PIIINP value for predicting fibrosis stage $\geq$ F1 was $242.3 \mathrm{ng} / \mathrm{mL}$, with $73.8 \%$ sensitivity and $90 \%$ specificity. The cut-off value for predicting cirrhosis was $698.7 \mathrm{ng} / \mathrm{mL}$, with $75 \%$ sensitivity and $96 \%$ specificity.

Conclusion: Serum PIIINP is a promising biomarker for predicting liver fibrosis stage, especially cirrhosis. Its assessment is a simple and non-invasive diagnostic method for liver fibrosis in infants with cholestasis.

Keywords: cirrhosis, liver fibrosis, infant, procollagen III N-terminal peptide, biliary atresia 


\section{INTRODUCTION}

Cholestasis is a central key manifestation of hepatobiliary disease in patients of all ages. The neonatal liver is more susceptible to cholestasis compared to older children and adults (1). Common causes of infantile cholestasis include biliary atresia, neonatal hepatitis (of which there are many etiologies), choledochal cyst, and other causes. Liver fibrosis is the common pathologic process of all chronic liver diseases, which results from excessive extracellular matrix accumulation (2-6). If effective treatment is not administered promptly, affected children can rapidly develop end-stage liver disease (cirrhosis, liver failure, and eventual death) in the first year of life (7). It is extremely important to evaluate liver fibrosis promptly to guide the clinical diagnosis and treatment and to improve prognosis.

The gold standard for assessment of fibrosis is liver biopsy and it is helpful for identifying the cause of cholestasis and guiding the treatment. However, in some areas, several issues limit its application for monitoring disease course, including risk of injury to the patient, variable accessibility of the damaged section of the liver, sampling errors, and inaccuracy due to interand intra-observer variability of pathologic interpretations (8). As a consequence, non-invasive and simple tools to accurately monitor disease course are highly desirable.

In previous studies, several non-invasive markers such as serum laminin (LN), hyaluronic acid (HA), and procollagen III $\mathrm{N}$-terminal peptide (PIIINP) have been reported to be indicators of liver fibrosis in adults with chronic viral hepatitis (9-11). However, the clinical value of these markers in infants with cholestasis remains unknown.

In the present study, we evaluated the clinical significance of serum LN, HA, and PIIINP in diagnosing liver fibrosis in infants with cholestasis.

\section{MATERIALS AND METHODS}

\section{Patients}

All term infants with cholestasis admitted to Xinhua Hospital Shanghai Jiao Tong University School of Medicine who underwent liver biopsy between July 2016 and July 2019 were enrolled in the present study. Cholestasis was defined as a value of direct bilirubin $<1.0 \mathrm{mg} / \mathrm{dL}$ if the total bilirubin is $<5 \mathrm{mg} / \mathrm{dL}$, or a value of direct bilirubin that represents more than $20 \%$ of the total bilirubin if the total bilirubin is $<5 \mathrm{mg} / \mathrm{dL}$ (12). The inclusion criteria were: (a) age between 1 week and 5 months; (b) diagnosis of cholestasis; and (c) availability of detailed clinical, imaging, and laboratory examination data. The exclusion criteria were: (a) low birth weight $(<2,500 \mathrm{~g})$; (b) previous administration of parenteral nutrition; (c) previous acute liver failure, shock, or sepsis; and (d) previous hepatobiliary surgery.

Abdominal ultrasonography, hepatobiliary scintigraphy, and magnetic resonance cholangiopancreatography were routine examinations administered to infants with cholestasis. Laparoscopic exploration and cholangiography were performed to diagnose or rule out biliary atresia when biliary atresia was highly suspected as indicated by imaging examinations, or persistent severe cholestasis was unresponsive to standard medical treatment and biliary atresia could not be ruled out. Biliary atresia was diagnosed when there was obliteration of extrahepatic bile ducts confirmed by cholangiography $(13,14)$. Kasai surgery was performed in infants with biliary atresia diagnosed by cholangiography, and bile duct irrigation was performed in infants without biliary atresia. Choledochal cyst resection and Roux-en-Y hepaticojejunostomy were performed in infants with choledochal cyst.

\section{Ethical Considerations}

This study was approved by the Ethics Committee of Xinhua Hospital Shanghai Jiao Tong University School of Medicine. The parents of the infants investigated in this study provided written informed consent for their infants' participation.

\section{Laboratory Detection}

Blood samples were obtained from all patients via a routine laboratory check-up prior to laparoscopic exploration. The blood cell counts were determined by automatic blood cell analysis. Serum aspartate aminotransferase (AST), alanine aminotransferase (ALT), gamma-glutamyl transferase (GGT), total bilirubin (TB), and direct bilirubin (DB) were measured using kits supplied by FUJIFILM Wako Pure Chemical Corporation, Osaka, Japan. Serum LN, HA, and PIIINP were quantified using chemiluminescent immunoassay. The kits were supplied by Shenzhen New Industry Biomedical Engineering Company, Shenzhen, China. The procedures were performed according to the manual.

\section{Liver Histology}

All patients underwent laparoscopic exploration and cholangiography, and liver biopsy was performed during laparotomy or laparoscopy. Wedge-shaped tissue was taken from the edge of one lobe of liver. The specimens were routinely formalin-fixed, paraffin-embedded, and stained with hematoxylin-eosin and Masson's trichrome stains. Samples containing at least six portal tracts were defined as adequate. Liver fibrosis was semi-quantitatively evaluated using the METAVIR scoring system (15). Fibrosis (F) was staged on a five-point scale (F0, no fibrosis; F1, portal fibrosis without septa; F2, portal fibrosis and few septa; F3, numerous septa without cirrhosis; F4, cirrhosis). Where there was evidence of more than one stage, the more severe stage was adopted for the analysis. The pathologist was blinded to the results of serum indices of the study subjects.

\section{Statistical Analyses}

Statistical analyses were performed using SPSS version 23.0 for Windows (SPSS Inc., Chicago, IL, USA). Continuous variables were summarized as mean \pm standard deviation or median and interquartile range (IQR) (25th and 75th percentile), depending on whether their distributions were normal, whereas categorical variables were summarized as numbers of cases and percentages. Demographic characteristics and laboratory examination results were compared between the fibrosis stages using the KruskalWallis test, chi-square test or Mann-Whitney $U$-test, as appropriate. Correlations between the stage of histological liver 
fibrosis and serum indices were assessed using a Spearman analysis. Multivariate ordinal regression analysis was performed with fibrosis stage vs. the parameters significantly correlated in the Spearman analysis for identification of independent predictors. Sensitivity of the assays was plotted against false positivity (1-specificity) using receiver operator characteristic (ROC) curves. Tests were considered statistically significant at $P<0.05$.

\section{RESULTS}

\section{Characteristics of the Patients}

From July 2016 to July 2019, 143 term infants with cholestasis were included, while one infant was excluded because of low birth weight, one because of parenteral nutrition, two because of shock or sepsis, and two because of previous hepatobiliary surgery. Therefore, 137 infants were eligible for statistical analysis, including 74 females (54.0\%) and 63 males (46.0\%), with a median (IQR) age of 54.0 (42.0-67.0) days. Ninety infants $(65.7 \%)$ were diagnosed with biliary atresia (four combined with cytomegalovirus infection), 36 (26.3\%) with choledochal cyst (one combined with cytomegalovirus infection), four $(2.9 \%)$ with cytomegalovirus hepatitis. One infant was diagnosed with Alagille syndrome with de novo heterozygous mutation (c.532delC) in JAG1. One infant was diagnosed with Zellweger syndrome with two heterozygous mutations in PEX26. One infant was diagnosed with $\mathrm{COACH}$ syndrome with two heterozygous mutations in CC2D2A. The other four infants with cholestasis were of indeterminate cause. According to the METAVIR scoring system, there were 30 F0 stage infants, 41 F1 stage infants, 26 F2 stage infants, 28 F3 stage infants, and 12 F4 stage infants.

\section{Comparisons of Parameters Between the Patients With Different Fibrosis Stages}

Table 1 shows the comparison of clinical characteristics and laboratory examination results in patients with different fibrosis stages. There were no statistically significant differences in gender and weight between patients of different fibrosis stages. Age tended to increase with the severity of fibrosis, especially in infants with cirrhosis $(P<0.05)$. The proportion of biliary atresia was higher in infants with higher fibrosis stage $(P<0.05)$. Serum levels of ALT, AST, TB, DB, GGT, PIIINP, and HA were significantly different in infants at different fibrosis stages $(P<$ $0.05)$. There was no significant difference in blood platelet count and serum LN level between any of the groups $(P>0.05)$.

The Spearman analysis showed a positive correlation between serum ALT, AST, TB, DB, GGT, PIIINP, and HA level and liver fibrosis stage $(r=0.409,0.446,0.299,0.471,0.247,0.622$, and 0.41 , respectively, $P<0.005)$, but there was no significant correlation between serum LN level and fibrosis stage $(P>$ 0.05). The Mann-Whitney $U$-test showed that serum HA level increased more in F2/F3/F4 than in F0/F1 (189.6 [139.0-282.1] vs. 111.5 [83.1-185.7] ng/mL, $Z=-4.64, P<0.001)$.

Multivariate ordinal regression analysis showed that serum AST, TB, DB, and PIIINP were independently correlated with fibrosis stage adjusted for age and biliary atresia, as shown in Table 2. Box-plot representation of serum TB, DB, AST, and PIIINP levels in infants at different fibrosis stages are shown in Figure 1.

\section{Diagnostic Performance of Indices Comparison}

ROC analysis revealed that serum PIIINP was the best diagnostic indicator of the stage of fibrosis, with an area under the curve

TABLE 1 | Comparison of characteristics in patients at different fibrosis stages.

\begin{tabular}{|c|c|c|c|c|c|c|}
\hline Characteristics & Fo & $\mathbf{F 1}$ & F2 & F3 & F4 & $P$-value \\
\hline Number & 30 & 41 & 26 & 28 & 12 & \\
\hline Age (days) ${ }^{\#}$ & $52.0(35.8,73.8)$ & $48.0(37.0,59.5)$ & $53.5(32.8,62.8)$ & $57.0(46.5,74.0)$ & $68.0(55.5,111.5)$ & $0.008^{\star}$ \\
\hline Males, n (\%) & $13(43.3)$ & $17(41.5)$ & $14(53.8)$ & $13(46.4)$ & $6(50.0)$ & 0.886 \\
\hline Weight $(g)^{\#}$ & $4850(3650,5425)$ & $4500(4250,5055)$ & $5000(3938,5525)$ & $5000(4350,5775)$ & $5150(4575,6325)$ & 0.295 \\
\hline $\mathrm{BA}, \mathrm{n}(\%)$ & 8 (26.7) & $25(61.0)$ & $22(84.6)$ & 25 (89.3) & 10 (83.3) & $<0.001^{\star}$ \\
\hline $\operatorname{ALT}(\mathrm{U} / \mathrm{L})^{\#}$ & $66.0(31.0,107.8)$ & $88.0(24.5,161.0)$ & $83.5(48.5,153.0)$ & $180.5(88.0,243.5)$ & $207.5(154.3,416.5)$ & $<0.001^{\star}$ \\
\hline AST $(U / L)^{\#}$ & $88.0(50.8,176.8)$ & $135.0(55.5,206.5)$ & $141.0(102.8,249.3)$ & $208.0(155.8,355.5)$ & $360.5(213.8,620.8)$ & $<0.001^{*}$ \\
\hline TB $(\mu \mathrm{mol} / \mathrm{L})^{\#}$ & $86.6(19.5,147.7)$ & $126.7(106.0,160.2)$ & $144.2(109.3,167.3)$ & $146.7(116.7,167.8)$ & $149.8(130.5,170.9)$ & $0.008^{\star}$ \\
\hline $\mathrm{DB}(\mu \mathrm{mol} / \mathrm{L})^{\#}$ & $14.2(5.0,86.5)$ & $81.8(24.4,97.4)$ & $83.7(71.9,117.9)$ & $102.9(85.1,120.7)$ & $111.6(84.8,124.5)$ & $<0.001^{*}$ \\
\hline $\mathrm{GGT}(\mathrm{U} / \mathrm{L})^{\#}$ & $268.0(94.0,652.8)$ & $207.0(132.5,489.0)$ & $463.5(250.0,736.3)$ & $405.0(237.5,577.8)$ & $523.0(212.3,1084.0)$ & $0.042^{\star}$ \\
\hline $\mathrm{BPC}\left(\times 10^{9} / \mathrm{L}\right)^{\#}$ & $368.0(293.5,528.8)$ & $352.0(268.0,485.0)$ & $403.5(201.3,539.5)$ & $387.5(314.0,573.8)$ & $350.0(213.8,449.3)$ & 0.747 \\
\hline LN (ng/mL) & $289.8(197.1,384.8)$ & $367.4(181.9,525.0)$ & $252.7(137.8,426.4)$ & $321.0(136.6,576.4)$ & $364.8(155.4,537.3)$ & 0.557 \\
\hline $\mathrm{HA}(\mathrm{ng} / \mathrm{mL})^{\#}$ & $109.7(78.4,132.2)$ & $112.0(88.7,207.0)$ & $180.2(135.8,242.7)$ & $161.3(122.3,214.4)$ & $296.8(206.7,547.0)$ & $<0.001^{*}$ \\
\hline PIIINP (ng/mL) & $170.9(110.9,206.9)$ & $273.7(178.4,371.9)$ & $274.9(210.4,497.1)$ & $363.2(324.5,473.2)$ & $811.9(483.7,985.2)$ & $<0.001^{*}$ \\
\hline
\end{tabular}

\# Results are presented as median and interquartile range (IQR, 25th and 75th percentile), because their distributions are not normal. Significant differences were evaluated using the Kruskal-Wallis test or Chi-square test.

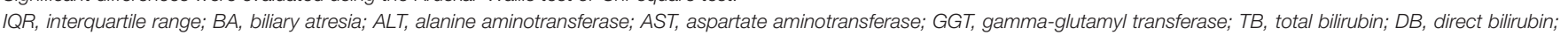
BPC, blood platelet count; LN, laminin; HA, hyaluronic acid; PIIINP, procollagen III N-terminal peptide.

*indicates significant difference. 
of $0.843,0.789,0.82$, and 0.891 for diagnosis of stages $\geq F 1, \geq F 2$, $\geq F 3$, and F4, respectively. More details are shown in Table 3 and

Figure 2. The cut-off values were set as the levels that resulted

TABLE 2 | Multivariate ordinal regression analysis for serum AST, TB, DB, and PIIINP with fibrosis stage after adjusting for age and biliary atresia.

\begin{tabular}{lcc}
\hline Parameter & Adjusted OR $\mathbf{( 9 5 \% ~ C l )}$ & $\boldsymbol{P}$-value \\
\hline ALT & $1.0025(0.9996-1.0055)$ & 0.09 \\
AST & $1.0024(1.0004-1.0045)$ & $0.021^{*}$ \\
TB & $1.0083(1.0023-1.0143)$ & $0.006^{*}$ \\
DB & $1.0153(1.0062-1.0245)$ & $0.001^{*}$ \\
GGT & $1.0003(0.9997-1.001)$ & 0.316 \\
HA & $1.0001(0.9997-1.0005)$ & 0.653 \\
PIIINP & $1.0054(1.0035-1.0073)$ & $<0.001^{*}$ \\
\hline
\end{tabular}

$O R$, odds ratio; $\mathrm{Cl}$, confidence interval; $A L T$, alanine aminotransferase; $A S T$, aspartate aminotransferase; GGT, gamma-glutamyl transferase; $T B$, total bilirubin; $D B$, direct bilirubin; LN, laminin; HA, hyaluronic acid; PIIINP, procollagen III N-terminal peptide. *indicates significant difference. in the maximum sum of sensitivity and specificity. The cut-off serum PIIINP value for predicting stages $\geq \mathrm{F} 1$ was $242.3 \mathrm{ng} / \mathrm{mL}$ with $73.8 \%$ sensitivity and $90 \%$ specificity, whereas the cut-off value for predicting F4 was $698.7 \mathrm{ng} / \mathrm{mL}$ with $75 \%$ sensitivity and $96 \%$ specificity.

\section{DISCUSSION}

In the present study, we found that serum PIIINP was the best diagnostic indicator of the stage of fibrosis in infants with cholestasis. As far as we know, this is the first study to evaluate serum PIIINP for liver fibrosis in infants.

In newborns and infants, congenital biliary atresia, choledochal cyst, and long-term cholestasis cause liver fibrosis (even cirrhosis) and may result in serious liver dysfunction. The severity of liver fibrosis is known to be a good indicator for determining the prognosis and optimal treatment of cholestasis $(16,17)$. Laparoscopic exploration should be performed as early as possible in infants with
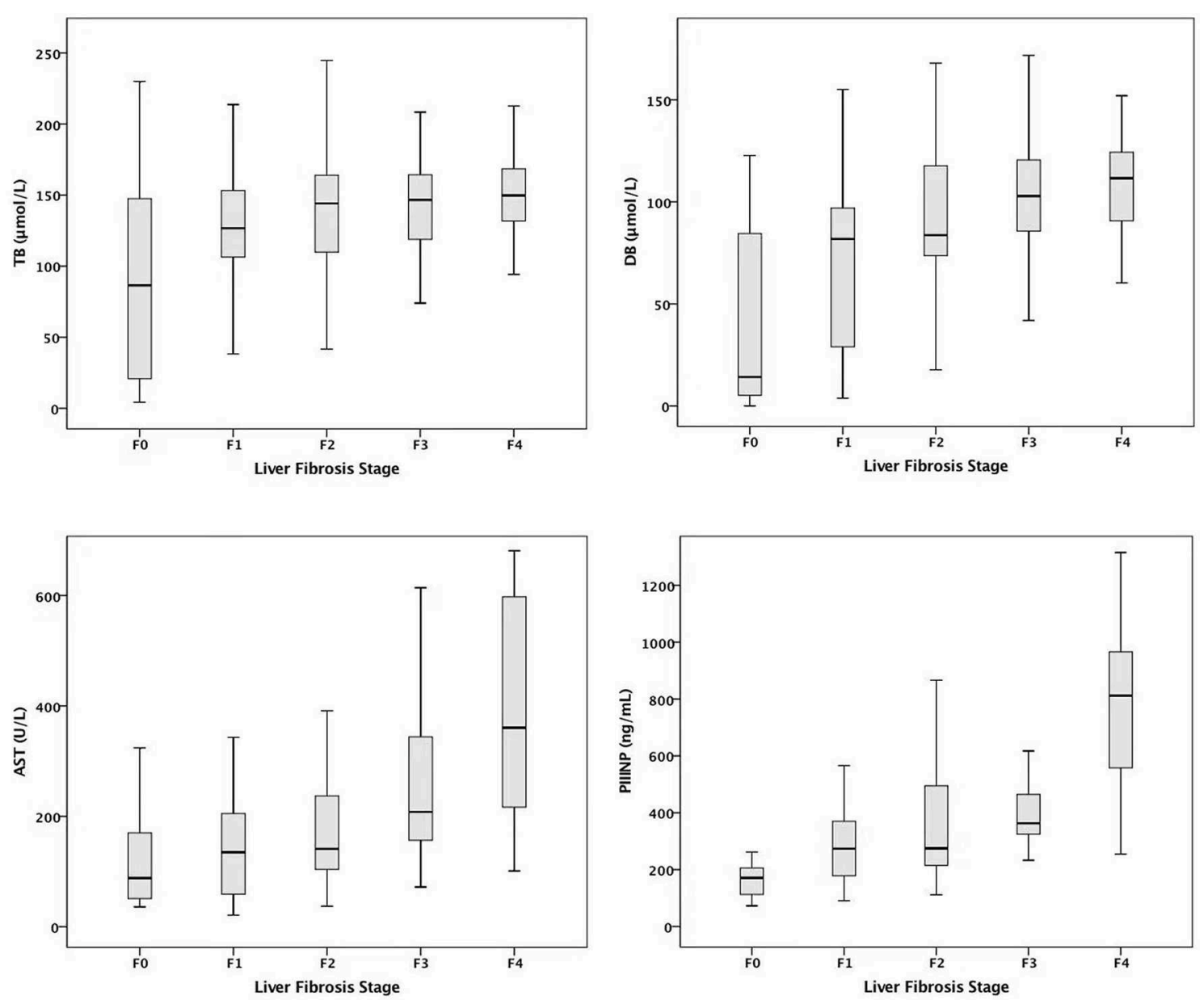

FIGURE 1 | Serum TB, DB, AST, and PIIINP levels in infants at different fibrosis stages. AST, aspartate aminotransferase; TB, total bilirubin; DB, direct bilirubin; PIIINP, procollagen III N-terminal peptide. 
TABLE 3 | Diagnostic performance of serum AST, TB, DB, and PIIINP for staging fibrosis grades.

\begin{tabular}{|c|c|c|c|c|}
\hline Indices & \multicolumn{4}{|c|}{ AUROC $(95 \%$ Cl) } \\
\hline TB & $0.7(0.576-0.824)$ & $0.648(0.555-0.74)$ & $0.617(0.522-0.711)$ & $0.635(0.506-0.765)$ \\
\hline DB & $0.746(0.638-0.854)$ & $0.737(0.655-0.82)$ & $0.746(0.662-0.83)$ & $0.739(0.605-0.872$ \\
\hline PIIINP & $0.843(0.766-0.92)$ & $0.789(0.714-0.864)$ & $0.82(0.752-0.889)$ & $0.891(0.788-0.995$ \\
\hline
\end{tabular}

AUROC, area under receiver operating curve; Cl, confidence interval; AST, aspartate aminotransferase; TB, total bilirubin; DB, direct bilirubin; PIIINP, procollagen III N-terminal peptide.
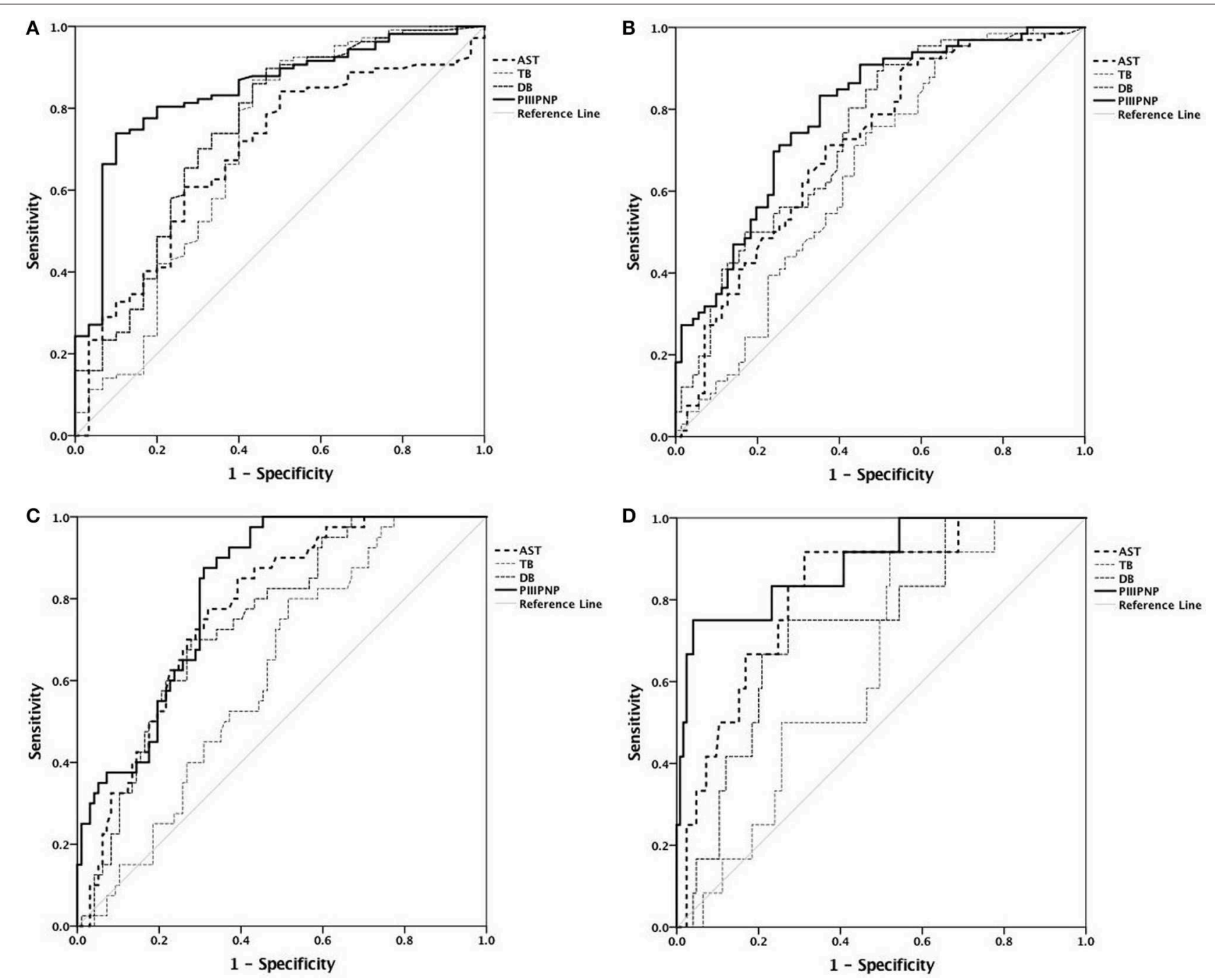

FIGURE 2 | Receiver operating characteristic curves. (A) Diagnosis of stage $\geq F 1$; (B) diagnosis of stage $\geq F 2$; (C) diagnosis of stage $\geq F 3$; (D) diagnosis of stage F4.

increasing fibrosis, if biliary atresia cannot be ruled out. In addition, liver fibrosis evaluation after surgery is helpful to evaluate the condition of the liver, and can guide the following treatment $(18,19)$.

Advanced chronic liver disease has been known to be accompanied by thrombocytopenia (20). A negative correlation has been reported to exist between the stage of liver fibrosis and blood platelet count, especially in patients with hepatitis $\mathrm{C}$ and non-alcoholic fatty liver disease (21). The platelet count has been used in scoring systems used for various liver diseases (22). However, in this study, there was no relationship between blood platelet count and liver fibrosis. This may 
because the infants in this study seldom had portal hypertension and enlargement of the spleen due to the short duration of cholestasis.

Cholestasis of different etiologies can cause different degrees of liver injury, manifested as abnormal liver function. Generally, liver function is more impaired with progression of the disease. ALT, AST, bilirubin, and GGT are well-known predictors of liver disease progression, reflecting the severity of hepatocellular injury and cholestasis. Our results showed that serum ALT, AST, TB, DB, and GGT were all significantly positively correlated with liver fibrosis stage. However, multivariate analysis showed that serum ALT and GGT were not independently associated with fibrosis stage when adjusted for age and biliary atresia. Mansoor et al. reported that the AST/ALT ratio and the AST to platelet ratio index (APRI), which are used to identify advanced fibrosis in adult patients, may not be accurate for predicting advanced fibrosis in children $(23,24)$. In our study, AST showed good diagnostic performance for cirrhosis (F4) with an AUROC of 0.822 . This may be because the increased serum AST may reflect hepatocyte inflammation, damage, or cholestasis that is related to the occurrence and progression of liver fibrosis.

$\mathrm{LN}$ is one of the main glycoproteins of the basement membrane and it is synthesized by hepatocytes and sinusoidal cells. Serum LN levels have shown a close positive and linear correlation with liver fibrosis stage in adults with chronic hepatitis $(25,26)$. In our study, there was no significant difference between infants at different fibrosis stages, and there was no significant correlation between serum LN level and fibrosis stage. The deposition of LN may result in sinusoid capillarization, which has a major correlation with portal hypertension (27). Kong et al. conducted a study on 39 adults with cirrhosis of various causes, and the results showed that serum LN levels decreased markedly immediately after splenectomy compared to pre-surgery, suggesting that LN may be mainly related to portal hypertension rather than liver fibrosis (28).

HA is a glycosaminoglycan synthesized by hepatic stellate cells (HSCs) and it is a component of the extra-cellular matrix (ECM). Li et al. reported that serum HA showed positive correlation with liver fibrosis stage in adults with chronic hepatitis B (26). Matsue et al. found that HA was effective in differentiating between F0/F1 and F2/F3/F4 in chronic hepatitis C patients (29). Similarly, in our study, serum HA levels increased more in F2/F3/F4 patients compared to F0/F1 patients. However, multivariate analysis showed that serum HA level was not independently associated with the fibrosis stage. In the study by Valva et al. HA was only related to worse fibrosis stages in adults with chronic hepatitis $\mathrm{C}$, but not in children (30).

PIIINP is a cleavage product of collagen precursor and has been the most closely studied non-invasive marker of liver fibrosis in chronic hepatitis $\mathrm{C}$ and primary biliary cirrhosis $(10,31)$. Many studies have showed a significant correlation between serum PIIINP level and histological liver fibrosis stage in adults (32-36). Leroy et al. (37) reported that serum PIIINP level was a good test of liver fibrosis stage in patients with chronic hepatitis $\mathrm{C}$, with an AUROC value of 0.88 for diagnosis of stages $\geq$ F3. Our results showed that serum PIIINP level increased significantly with the severity of liver fibrosis, and the correlation between serum PIIINP level and liver fibrosis stage was strong, positive, and linear. Serum PIIINP was an independent predictor of liver fibrosis stage by multivariate ordinal regression analysis. These findings suggest that infants with higher serum PIIINP levels might have a higher liver fibrosis stage, which is compatible with other reports in the literature regarding adults.

ROC analysis showed that serum PIIINP was of a moderate diagnostic value for fibrosis stage, and better than serum AST, TB, and DB. Serum PIIINP was most accurate for diagnosing cirrhosis, with the largest AUROC of 0.891 . While the diagnosis of $\geq F 1$ came second with an AUROC of 0.843 , the accuracy was poorest for diagnosis of stages $\geq F 2$ with an AUROC of 0.789 . These results indicated that serum PIIINP was more useful for detecting liver fibrosis and cirrhosis than discriminating between mild and moderate fibrosis. We identified serum PIIINP cutoff values as $242.3 \mathrm{ng} / \mathrm{mL}$ for predicting stages $\geq \mathrm{F} 1$ and $698.7 \mathrm{ng} / \mathrm{mL}$ for predicting cirrhosis.

\section{Limitations}

Our sample size was relatively limited and infants with different diseases were included in the study. Thus, we do not believe that serum PIIINP can replace liver biopsy for the assessment of liver fibrosis at this point in time. Further prospective studies are required to determine the potential utility of serum PIIINP testing in guiding treatment decisions and monitoring disease course.

\section{CONCLUSION}

Serum PIIINP level showed moderate diagnostic value as an independent predictor of liver fibrosis stage. Its assessment is a simple and non-invasive diagnostic method and it is a promising biomarker for predicting liver fibrosis stage, especially cirrhosis in infants with cholestasis.

\section{DATA AVAILABILITY STATEMENT}

The datasets generated for this study are available on request to the corresponding author.

\section{ETHICS STATEMENT}

The studies involving human participants were reviewed and approved by Ethics Committee of Xin Hua Hospital Affiliated to Shanghai Jiao Tong University School of Medicine. Written informed consent to participate in this study was provided by the participants' legal guardian/next of kin.

\section{AUTHOR CONTRIBUTIONS}

YW contributed to the acquisition, analysis, and interpretation of data for the work. WP performed the operations and 
approved the version to be submitted. DZ and YC contributed to analysis and interpretation of the data. XC contributed to liver fibrosis evaluation. HX contributed to the design of the work and revised the paper critically for important intellectual content. All authors have read and approved the final version to be published.

\section{REFERENCES}

1. Talachian E, Bidari A, Mehrazma M, Nick-khah N. Biopsy-driven diagnosis in infants with cholestatic jaundice in Iran. World J Gastroenterol. (2014) 20:1048-53. doi: 10.3748/wjg.v20.i4.1048

2. Benyon RC, Iredale JP. Is liver fibrosis reversible? Gut. (2000) 46:4436. doi: 10.1136/gut.46.4.443

3. Amy GF, Cara LM. Biliary atresia: clinical lessons learned. J Pediatr Gastroenterol Nutr. (2015) 61:167-75. doi: 10.1097/MPG.0000000000000755

4. Bataller R, Brenner DA. Liver fibrosis. J Clin Invest. (2005) 115:20918. doi: $10.1172 /$ JCI24282

5. Friedman SL. Mechanisms of hepatic fibrogenesis. Gastroenterology. (2008) 134:1655-69. doi: 10.1053/j.gastro.2008.03.003

6. Bae M, Park YK, Lee JY. Food components with anti-fibrotic activity and implications in prevention of liver disease. J Nutr Biochem. (2018) 55:111. doi: 10.1016/j.jnutbio.2017.11.003

7. Hung PY, Chen CC, Chen WJ, Lai HS, Hsu WM, Lee PH, et al. Long-term prognosis of patients with biliary atresia: a 25 year summary. J Pediatr Gastroenterol Nutr. (2006) 42:1905. doi: 10.1097/01.mpg.0000189339.92891.64

8. Shiha G, Ibrahim A, Helmy A, Sarin SK, Omata M, Kumar A, et al. Asian-Pacific association for the study of the liver (APASL) consensus guidelines on invasive and non-invasive assessment of hepatic fibrosis: a 2016 update. Hepatol Int. (2016) 11:1-30. doi: 10.1007/s12072-0169760-3

9. Guéchot J, Serfaty L, Bonnand AM, Chazouillères O, Poupon RE, Poupon R. Prognostic value of serum hyaluronan in patients with compensated HCV cirrhosis. J Hepat. (2000) 32:447-52. doi: 10.1016/S0168-8278(00) 80396-7

10. Leroy V, De Traversay C, Barnoud R, Hartmann JD, Baud M, Ouzan D, et al. Changes in histological lesions and serum fibrogenesis markers in chronic hepatitis C patients non-responders to interferon alpha. J Hepat. (2001) 35:120-6. doi: 10.1016/S0168-8278(01)00091-5

11. Castera L. Non-invasive methods to assess liver disease in patients with hepatitis B or C. Gastroenterology. (2012) 142:1293302. doi: 10.1053/j.gastro.2012.02.017

12. Moyer V, Freese DK, Whitington PF, Olson AD, Brewer F, Colletti $\mathrm{RB}$, et al. Guideline for the evaluation of cholestatic jaundice in infants: recommendations of the North American society for pediatric gastroenterology, hepatology and nutrition. $J$ Pediatr Gastroenterol Nutr. (2004) 39:115-28. doi: 10.1097/00005176-20040800000001

13. Fischler B, Haglund B, Hjern A. A population-based study on the incidence and possible pre- and perinatal etiologic risk factors of biliary atresia. J. Pediatr. (2002) 141:217-22. doi: 10.1067/mpd.2002.126001

14. McKiernan PJ, Baker AJ, Kelly DA. The frequency and outcome of biliary atresia in the UK and Ireland. Lancet. (2000) 355:259. doi: 10.1016/S0140-6736(99)03492-3

15. Bedossa P, Poynard T. An algorithm for the grading of activity in chronic hepatitis C. metavir cooperative study group. Hepatology. (1996) 24:28993. doi: 10.1002/hep.510240201

16. Fawaz R, Baumann U, Ekong U, Fischler B, Hadzic N, Mack CL, et al. Guideline for the evaluation of cholestatic jaundice in infants: joint recommendations of the North American society for pediatric gastroenterology, hepatology, and nutrition and the European society for pediatric gastroenterology, hepatology, and nutrition. J Pediatr Gastroenterol Nutr. (2017) 64:154-68. doi: 10.1097/MPG.0000000000001334

\section{FUNDING}

This work was supported by grants from Shanghai Municipal Commission of Health and Family Planning (grant no. 2016ZB0103) and the National Natural Science Foundation of China (grant no. 81200458).

17. Sharma S, Das P, Dattagupta S, Kumar L, Gupta DK. Liver and portal histopathological correlation with age and survival in extra hepatic biliary atresia. Pediatr Surg Int. (2011) 27:451-61. doi: 10.1007/s00383-010-2845-5

18. Mukhopadhyay SG, Roy P, Chatterjee U, Datta C, Banerjee M, Banerjee S, et al. A histopathological study of liver and biliary remnants in the long-term survivors (> 10 years) of cases of biliary atresia. Indian J Pathol Micr. (2014) 57:380-5. doi: 10.4103/0377-4929. 138722

19. Russo P, Magee JC, Anders RA, Bove KE, Chung C, Cummings OW, et al. Key histopathological features of liver biopsies that distinguish biliary atresia from other causes of infantile cholestasis and their correlation with outcome: a multicenter study. Am J Surg Pathol. (2016) 40:160115. doi: 10.1097/PAS.0000000000000755

20. Poynard T, Yuen MF, Ratziu V, Lai CL. Viral hepatitis C. Lancet. (2003) 362:2095-100. doi: 10.1016/S0140-6736(03)15109-4

21. Kajihara M, Okazaki Y, Kato S, Ishii H, Kawakami Y, Ikeda Y, et al. Evaluation of platelet kinetics in patients with liver cirrhosis: similarity to idiopathic thrombocytopenic purpura. J Gastroenterol Hepatol. (2007) 22:112-8. doi: 10.1111/j.1440-1746.2006.04359.x

22. Yoneda M, Fujii H, Sumida Y, Hyogo H, Itoh Y, Ono M, et al. Platelet count for predicting fibrosis in non-alcoholic fatty liver disease. J Gastroenterol. (2011) 46:1300-6. doi: 10.1007/s00535-011-0436-4

23. Mansoor S, Yerian L, Kohli R, Xanthakos S, Angulo P, Ling S, et al. The evaluation of hepatic fibrosis scores in children with nonalcoholic fatty liver disease. Dig Dis Sci. (2015) 60:1440-7. doi: 10.1007/s10620-0143494-7

24. Mansoor S, Collyer E, Alkhouri N. A comprehensive review of noninvasive liver fibrosis tests in pediatric non-alcoholic Fatty liver disease. Curr Gastroenterol Rep. (2015) 17:23-9. doi: 10.1007/s11894-015-0447-Z

25. Parsian H, Rahimipour A, Nouri M, Somi MH, Qujeq D, Fard MK, et al. Serum hyaluronic acid and laminin as biomarkers in liver fibrosis. J Gastrointestin Liver Dis. (2010) 19:169-74. doi: 10.1111/j.1440-1746.2010.06363.x

26. Li F, Zhu CL, Zhang H, Huang H, Wei Q, Zhu X, et al. Role of hyaluronic acid and laminin as serum markers for predicting significant fibrosis in patients with chronic hepatitis B. Braz J Infect Dis. (2012) 16:914. doi: 10.1590/S1413-86702012000100002

27. Zhu C, Qi X, Li H, Peng Y, Dai J, Chen J, et al. Correlation of serum liver fibrosis markers with severity of liver dysfunction in liver cirrhosis: a retrospective cross-sectional study. Int J Clin Exp Med. (2015) 8:5989-98.

28. Kong D, Chen X, Lu S, Guo Q, Lai W, Wu J, et al. Short-term effects of splenectomy on serum fibrosis indexes in liver cirrhosis patients. Int J Clin Exp Pathol. (2015) 8:15260-4.

29. Matsue Y, Tsutsumi M, Hayashi N, Saito T, Tsuchishima M, Toshikuni N, et al. Serum osteopontin predicts degree of hepatic fibrosis and serves as a biomarker in patients with hepatitis C virus infection. PLoS ONE. (2015) 10:e0118744. doi: 10.1371/journal.pone.0118744

30. Valva P, Casciato P, Diaz Carrasco JM, Gadano A, Galdame O, Galoppo MC, et al. The role of serum biomarkers in predicting fibrosis progression in pediatric and adult hepatitis $\mathrm{C}$ virus chronic infection. PLoS ONE. (2011) 6:e23218. doi: 10.1371/journal.pone.00 23218

31. Shimizu I, Omoya T, Takaoka T, Wada S, Wada H, Taoka M, et al. Serum amino-terminal propeptide of type III procollagen and 7S domain of type IV collagen correlate with hepatic iron concentration in patients with chronic hepatitis C following $\alpha$-interferon therapy. J Gastroen Hepat. (2001) 16:196201. doi: 10.1046/j.1440-1746.2001.02402.x 
32. Zeng T, Yu J, Tan L, Wu Y, Tian Y, Wu Q, et al. Noninvasive indices for monitoring disease course in Chinese patients with autoimmune hepatitis. Clin Chim Acta. (2018) 486:135-41. doi: 10.1016/j.cca.2018.07.030

33. Murawaki Y, Ikuta Y, Okamoto K, Koda M, Kawasaki H. Diagnostic value of serum markers of connective tissue turnover for predicting histological staging and grading in patients with chronic hepatitis C. J Gastroen. (2001) 36:399-406. doi: 10.1007/s005350170084

34. Zheng M, Cai WM, Weng HL, Liu RH. ROC curves in evaluation of serum fibrosis indices for hepatic fibrosis. World J Gastroenterol. (2002) 8:10736. doi: 10.3748/wjg.v8.i6.1073

35. Patel K, Gordon SC, Jacobson I, Hézode C, Oh E, Smith KM, et al. Evaluation of a panel of non-invasive serum markers to differentiate mild from moderateto-advanced liver fibrosis in chronic hepatitis C patients. J Hepat. (2004) 41:935-42. doi: 10.1016/j.jhep.2004.08.008

36. Wong GL, Chan HL, Choi PC, Chan AW, Yu Z, Lai JW, et al. Non-invasive algorithm of enhanced liver fibrosis and liver stiffness measurement with transient elastography for advanced liver fibrosis in chronic hepatitis B. Aliment Pharm Therap. (2014) 39:197-208. doi: 10.1111/apt.12559
37. Leroy V, Monier F, Bottari S, Trocme C, Sturm N, Hilleret MN et al. Circulating matrix metalloproteinases 1, 2, 9 and their inhibitors TIMP-1 and TIMP-2 as serum markers of liver fibrosis in patients with chronic hepatitis C: comparison with PIIINP and hyaluronic acid. Am J Gastroenterol. (2004) 99:271-9. doi: 10.1111/j.1572-0241.2004. 04055.x

Conflict of Interest: The authors declare that the research was conducted in the absence of any commercial or financial relationships that could be construed as a potential conflict of interest.

Copyright (c) 2020 Wang, Pan, Zhao, Chen, Chen and Xia. This is an open-access article distributed under the terms of the Creative Commons Attribution License (CC $B Y)$. The use, distribution or reproduction in other forums is permitted, provided the original author(s) and the copyright owner(s) are credited and that the original publication in this journal is cited, in accordance with accepted academic practice. No use, distribution or reproduction is permitted which does not comply with these terms. 